\title{
Um perfil da produção científica de enfermagem em Hematologia, Hemoterapia e Transplante de medula óssea
}

\author{
Trends of nursery scientific output in Hematology, Hemotherapy and bone marrow Transplant \\ Estudio de la producción cientifica de enfermería en Hematologia, Hemoterapia y Transplante de \\ médula ósea
}

\section{Kizi Mendonça de Araújo', Marcos Antonio Gomes Brandão², Jacqueline Leta ${ }^{3}$}

\section{RESUMO}

Objetivos: Esse é um estudo exploratório transversal que pretendeu caracterizar a produção científica de enfermagem em Hematologia, Hemoterapia e Transplante de Medula Óssea, áreas de interesse crescente na enfermagem, por envolver situações que requerem cuidado qualificado e especializado do profissional desta área. Métodos: Foram analisados os resumos dos trabalhos publicados em três periódicos nacionais, em dois anais de congressos nacionais e da Biblioteca Virtual em Saúde, BVS/BIREME. Resultados: Do período 2000-2004, foram encontradas 88 publicações, a maior parte divulgada nos anais de congressos $(n=73)$. As categorias que mais contribuíram foram docentes e discentes $(n=40)$. Os trabalhos originaram-se principalmente no Sudeste $(n=49)$ e predominam aqueles com abordagem quantiqualitativa $(n=29)$ e na área assistencial $(n=51)$. Conclusões: Apesar das limitações, acreditamos que esse trabalho poderá estimular a discussão sobre a produção e divulgação científica de enfermagem nessas áreas.

Descritores: Bibliometria; Publicações científicas e técnicas; Publicações de divulgação científica; Enfermagem

\begin{abstract}
Obejctives: This transversal cohort study aims to portray the scientific production of nursery in hematology, hemotherapy and bone marrow transplant, fields where nursery involvement has grown steadily especially due to the fact that they deal with some patients' conditions that require qualified and specialized cares, characteristics found among nursery professionals. Methods: To describe the scientific output of the fields, we analyzed the abstracts published in three domestic periodicals, in two congress annals and the Virtual Library, BVS/BIREME. Results: In the 2000-2004 period, we found 88 publications; most were published in the annals ( $\mathrm{n}=73$ ). Professors and students were the professional categories that contributed the most $(n=40)$; institutions from the southeast region carried out 49 publications; publications with a quanti-qualitative approach summed up 29 while those related to assistance counted 51. Conclusions: Despite the limitations of this study, we believe that it may encourage the discussion about the nursery scientific output and diffusion in such fields.
\end{abstract}

Keywords: Bibliometrics; Scientific and technical publications; Publications for science diffusion; Nursing

\section{RESUMEN}

Objetivos: El presente estudio tiene como objetivo caracterizar la producción científica de enfermería en Hematología, Hemoterapia y Transplante de Médula Ósea. La participación de la enfermería en estos campos ha crecido continuamente debido a situaciones que requieren un cuidado especializado y cualificado de los profesionales de enfermería. Métodos: Para describir la producción científica en estos campos, analizamos resúmenes publicados en tres periódicos nacionales, en dos congresos anuales y en la Biblioteca Virtual BVS/BIREME. Resultados: Durante el periodo 2000-2004, encontramos 88 publicaciones, la mayor parte de ellas $(n=73)$ en los congresos anuales. Las categorías profesionales que más contribuyeron fueron profesores y estudiantes $(n=40)$. Los trabajos se originaron principalmente en el Sudeste $(n=49)$ y predominaron aquellos con un enfoque cuanti-cualitativo $(n=29)$ y los del área asistencial $(n=51)$. Conclusión: A pesar de las limitaciones, creemos que este trabajo podrá estimular una discusión sobre la producción y la divulgación científica de enfermería en esas áreas.

Descriptores: Bibliometría; Publicaciones científicas y técnicas; Publicaciones de divulgación científica; Enfermería

${ }^{1}$ Pós-graduanda em Educação, Gestão e Difusão em Biociências, Instituto de Bioquímica Médica, Universidade Federal do Rio de Janeiro - UFRJ - Rio de Janeiro (RJ), Brasil.

${ }^{2}$ Professor Assistente do Departamento de Enfermagem Fundamental, Pesquisador do Núcleo de Pesquisa de Fundamentos do Cuidado de Enfermagem , Universidade Federal do Rio de Janeiro - UFRJ - Rio de Janeiro (RJ), Brasil.

${ }^{3}$ Professora Adjunta do Programa de Educaşão, Gestão e Difusão em Biociências do Instituto de Bioquímica Médica - Universidade Federal do Rio de Janeiro - UFRJ - Rio de Janeiro (RJ), Brasil. 


\section{INTRODUÇÃO}

A produção cientifica em enfermagem teve como publicação-marco "Notes on Nursing", em 1859(1). Desde então, a publicação em enfermagem no mundo vem tornando-se crescente. Contudo, no Brasil a pesquisa em enfermagem e sua divulgação ainda são incipientes e têm demonstrado uma evolução lenta e gradual. Alguns marcos históricos, tal como a criação de programas de mestrado em diferentes Escolas de Enfermagem na década de 70, e de doutorado, no início dos anos 80, incentivaram a formação de novos pesquisadores e o desenvolvimento da pesquisa na área ${ }^{(2)}$.

Nos últimos anos, a procura pelos cursos de graduação em enfermagem vem crescendo significativamente em todo o país. Isso é possível observar através do aumento no total de cursos presenciais registrados no Ministério de Educação (MEC). Segundo dados do $\mathrm{MEC}^{(3)}$, de 2000 a 2002, os Programas e/ou Cursos de Graduação em Enfermagem e Enfermagem e Obstetrícia cresceram de 176 para 275. Este aumento, em tese, deveria se refletir também na produção cientifica, uma vez que o curso de enfermagem tem como diretriz "formar profissional qualificado para o exercício de enfermagem, com base no rigor científico e intelectual, pautado em princípios éticos". Além disso, os cursos de enfermagem devem assegurar: (a) a articulação entre o ensino, a pesquisa e a extensão/ assistência; (b) garantir um ensino crítico, reflexivo e criativo, que leve a construção do perfil almejado; (c) estimular a realização de experimentos e/ ou de projetos de pesquisa e (d) socializar o conhecimento produzido, levando em conta a evolução epistemológica dos modelos explicativos do processo saúde-doença ${ }^{(4)}$.

Apesar das diretrizes curriculares se relacionarem com a pesquisa científica, esta relação parece ainda incipiente. Um indicativo é o reduzido número de grupos de pesquisa na área de enfermagem no Diretório de Grupos de Pesquisa do $\mathrm{CNPq}^{(5)}$. Em 2002 haviam 2.513 grupos registrados na área de saúde em todo os pais, destes apenas 231, ou seja, 9,2\%, estavam registrados na enfermagem.

Diante disso, acreditamos que, conhecer o estado atual da pesquisa em enfermagem, pode contribuir para um melhor entendimento da área e auxiliar em políticas que visem estimular e desenvolver a maior interação no tripé pesquisa-ensino-assistencia, fundamental para a enfermagem. Considerando o quadro da pesquisa em enfermagem, delineado anteriormente, realizamos um recorte na área, e elegemos a produção científica em enfermagem hematológica como nosso objeto de estudo.

\section{A enfermagem hematológica}

A geração acelerada de novos conhecimentos torna necessária, cada vez mais, a produção de serviços e formação de profissionais especializados nas diferentes áreas do conhecimento. A crescente complexidade decorrente da especialização e da ampliação das conquistas tecnológicas na área da saúde também se reflete na enfermagem. Este processo tem levado os profissionais de enfermagem a buscar cursos de especialização em diferentes áreas, tal como na enfermagem hematológica. Essa área vem ganhando destaque e importância cientifica e acadêmica nos últimos anos por estar envolvida com uma série de doenças que são cada vez mais freqüentes em nossas vidas, como exemplo, a leucemia. Dados do Instituto Nacional do Câncer ${ }^{(6)}$ registram que as regiões Sul e Sudeste e alguns estados da região Nordeste, tal como Pernambuco, são aquelas com maiores índices de mortalidade por este tipo de câncer. Assim, dados epidemiológicos, como o apresentado, ilustram a magnitude do problema e apontam para a necessidade de formar profissionais de saúde (em especial na enfermagem) qualificados para atuar nestas enfermidades.

Ainda que possamos encontrar informações sobre a prevalência dos diagnósticos de enfermagem relacionados aos distúrbios hematológicos, carecemos de informações sobre a pesquisa em enfermagem nesta área. Assim, tomamos esta premissa como ponto de partida para o questionamento: "Qual o perfil da produção científica de enfermagem na área de hematologia, hemoterapia e de transplante de medula óssea?" Diante deste questionamento, o presente estudo teve como objetivo apresentar e caracterizar o perfil da produção científica atual em enfermagem nas áreas de Hematologia, Hemoterapia e Transplante de Medula Óssea, denominadas HHTMO.

\section{MÉTODOS}

A idéia de utilizar a literatura científica como objeto de estudo remonta ao início do século XX. Os dados quantitativos compilados a partir da análise da literatura científica têm sido utilizados como ferramentas para uma série de propósitos, tais como as citadas por Narin et al. ${ }^{(7)}$, sendo: político, estratégico, planejamento e convencional. Além disso, a análise da literatura, científica seja por temas, sub-áreas ou instituições, oferece informações úteis e consistentes sobre desempenho e tendências, podendo apontar carências e competências do objeto em análise ${ }^{(8-9)}$. No presente estudo, a produção científica em enfermagem na área de HHTMO foi analisada por critérios quantitativos e qualitativos a partir do levantamento dos resumos das seguintes fontes literárias:

- Anais de Congressos: "Congresso Brasileiro de HHTMO" e "Congresso Brasileiro de Enfermagem".

- Periódicos Nacionais: "Revista Brasileira de Hematologia e Hemoterapia", "Revista Brasileira de Enfermagem" e "Revista Latino-Americana de Enfermagem".

- Biblioteca Virtual: Biblioteca Virtual em Saúde 
(BVS/BIREME).

A escolha destas fontes deveu-se ao fato desses eventos e periódicos estarem entre os de maior abrangência nacional das áreas de HHTMO e da Enfermagem como um todo. Os artigos dessas áreas foram selecionados manualmente, a partir dos títulos e/ou da categoria profissional dos autores. Os dados coletados referem-se ao período de 2000 a 2004 e foram organizados em um banco de dados, em formato $\mathrm{Excel}^{\mathrm{O}}$, contendo informações, tais como: o ano de publicação; o autor de referência; a instituição do autor; a região geográfica da produção; a área temática; a categoria profissional do autor; o tipo de publicação; e a metodologia utilizada. A classificação das áreas temáticas dos artigos segue aquela apresentada por Assis et al. ${ }^{(10)}$ que são as seguintes: assistencial (sistematização das ações de enfermagem); administrativa (organização, planejamento, direção, supervisão e controle da assistência de enfermagem); de ensino (relacionados ao ensino e aprendizagem de enfermagem); profissional (recursos humanos, mercado de trabalho, integração docente assistencial, divisão social do trabalho, políticas de saúde, ética, legislação, produção, divulgação e utilização do conhecimento em enfermagem); de reflexão teórica (desenvolvimento de testagem de teorias e conceitos de enfermagem e outras áreas, elaboração e testagem de instrumentos) e outros (trabalhos que não se enquadraram a nenhuma das classificações anteriores). Para a análise da categoria profissional, considerou-se apenas o primeiro autor do artigo, classificado como: aluno de graduação; aluno de pós-graduação; enfermeiro-assistencial; ou docente. Para classificar o tipo de publicação, utilizamos a classificação de Pincus et al. ${ }^{(11)}$, que propõe o enquadramento do estudo como: pesquisa; estudo de caso; revisão; ou outros. Quanto à metodologia, as publicações foram classificadas em três abordagens: a qualitativa, a quantitativa ou a quanti-qualitativa.

\section{RESULTADOS}

Evolução e distribuição das publicações segundo as fontes e abordagem metodológica

A análise do total de publicações de enfermagem indicou um total de 88 trabalhos publicados no período. Se considerarmos os dados referentes aos participantes dos grupos de pesquisa, assim como alunos e profissionais de enfermagem no país (Tabela 1), o número encontrado é relativamente baixo.

Como podemos observar na figura 1 , a produção e divulgação de trabalhos científicos são relativamente estáveis no período, apresentando oscilações para menos nos anos de 2001 e 2002. A relação entre a produção de trabalhos no ano de 2002 (13 trabalhos) e o número de pesquisadores de enfermagem em 2002 (1537 pesquisadores) foi de 01 trabalho para cada 96 pesquisadores de enfermagem.

Tabela 1 - Grupos e linhas de pesquisa cadastrados no Diretório de Grupos de Pesquisa do CNPq, 2002.

\begin{tabular}{lrrrrrrr}
\hline $\begin{array}{l}\text { Área de } \\
\text { conhecimento }\end{array}$ & Grupos & $\begin{array}{c}\text { Pesquisa } \\
\text { dores }\end{array}$ & $\begin{array}{c}\text { Estuda } \\
\text { ntes }\end{array}$ & $\begin{array}{r}\text { Técnic } \\
\text { os }\end{array}$ & $\begin{array}{c}\text { Linhas } \\
\text { de } \\
\text { pesquisa }\end{array}$ \\
\hline Educação Física & 196 & 855 & 877 & 92 & 506 \\
Enfermagem & $\mathbf{2 3 1}$ & $\mathbf{1 . 5 3 7}$ & $\mathbf{9 6 3}$ & $\mathbf{2 5 6}$ & $\mathbf{6 5 7}$ \\
Farmácia & 171 & 1.072 & 1.014 & 235 & 579 \\
$\begin{array}{l}\text { Fisioterapia e } \\
\text { Terapia Ocupacional }\end{array}$ & 61 & 289 & 278 & 59 & 186 \\
Fonoaudiologia & 66 & 347 & 335 & 35 & 158 \\
Medicina & 925 & 4.329 & 2.889 & 2.046 & 3.307 \\
Nutrição & 100 & 589 & 494 & 154 & 302 \\
Odontologia & 375 & 2.028 & 1.274 & 307 & 1.234 \\
Saúde Coletiva & 388 & 2.452 & 1.347 & 798 & 1.178 \\
\hline TOTAL & $\mathbf{2 . 5 1 3}$ & $\mathbf{1 3 . 4 9 8}$ & $\mathbf{9 . 4 7 1}$ & $\mathbf{3 . 9 8 2}$ & $\mathbf{8 . 1 0 7}$ \\
\hline
\end{tabular}

A situação se agrava quando acrescentarmos no cálculo a parcela de estudantes: 01 trabalho para cada 192 pesquisadores e estudantes envolvidos em pesquisa. É importante, no entanto, ressaltar que esta relação possui limitações ao se considerar o universo de especialidades na enfermagem. Em relação à distribuição dos trabalhos segundo a fonte de divulgação, constatamos um predomínio das apresentações em congressos, com 73 artigos, e apenas 15 artigos divulgados nos periódicos analisados (Figura 1).

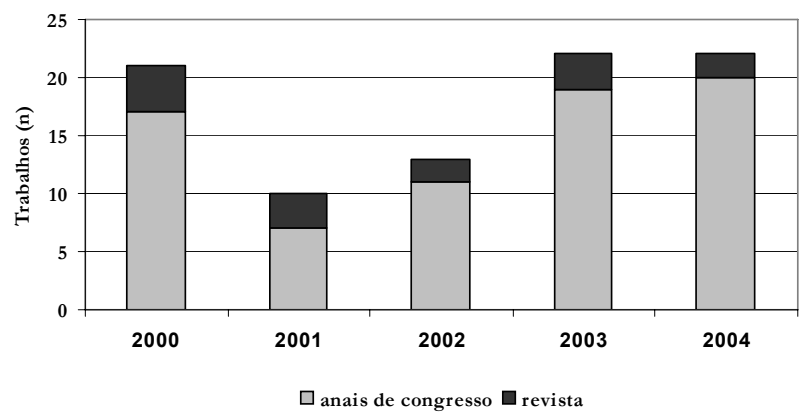

Figura 1 - Produção de enfermagem em HHTMO segundo a fonte de publicação no período de 20002004.

O desenho das publicações foi classificado de duas formas: tipo e abordagem metodológica (Tabela 2). Em relação ao tipo de trabalho apresentado, 54 dos 88 trabalhos $(60 \%)$ foram classificados como artigos de pesquisa; $17(20 \%)$ como outros; 11 (13\%) como estudo de caso; $06(7 \%)$ como revisão. Entre as publicações classificadas como "outros" há prevalência de relatos de experiência; 13 das 17 publicações. 
Tabela 2 - Distribuição da Produção de enfermagem em HHTMO quanto ao tipo e abordagem metodológica. Brasil, no período de 2000 a 2004.

\begin{tabular}{lcr}
\hline Tipo de Publicação & $\mathbf{f}$ & $\mathbf{0}$ \\
\hline Pesquisa & 54 & 60 \\
Outros & 17 & 20 \\
Estudo de caso & 11 & 13 \\
Revisão & 06 & 7 \\
\hline Total & $\mathbf{8 8}$ & $\mathbf{1 0 0}$ \\
\hline Abordagem Metodologia & & \\
\hline Quanti-qualitativa & 28 & 52 \\
Qualitativa & 22 & 41 \\
Quantitativa & 04 & 7 \\
\hline Total & $\mathbf{5 4}$ & $\mathbf{1 0 0}$ \\
\hline
\end{tabular}

\section{Distribuição das publicações segundo a área temática e categoria dos autores}

Quanto à abordagem metodológica, entre os artigos classificados como "pesquisa", observamos que 28 (52\%) trabalhos se enquadram em uma abordagem quantiqualitativa; $22(41 \%)$ na qualitativa e apenas $04(7 \%)$ na quantitativa (Tabela 2). A predominância por abordar os fenômenos e objetos de pesquisa a partir de uma perspectiva qualitativa pode ter origem em fatores diversos. Foge aos nossos propósitos, investigar essa questão em profundidade.

Das seis áreas temáticas utilizadas no estudo, a área predominante foi a assistencial com 51 publicações, em um total de 88, enquanto a área administrativa apresenta 03 publicações (Tabela 3).

Tabela 3 - Distribuição da Produção de enfermagem em HHTMO por área temática e categoria profissional, Brasil, 2000-2004.

\begin{tabular}{lrr}
\hline Área Temática & \multicolumn{1}{c}{ f } & \% \\
\hline Assistencial & 51 & 58 \\
Reflexão teórica & 14 & 16 \\
Profissional & 11 & 12 \\
Outros & 05 & 6 \\
Educação & 04 & 5 \\
Administrativa & 03 & 3 \\
\hline Total & $\mathbf{8 8}$ & $\mathbf{1 0 0}$ \\
\hline Categoria Profissional & & \\
\hline Enfermeiro / assistente & 18 & 31 \\
Discente (graduação/ pós) & 33 & 55 \\
Docente & 8 & 14 \\
\hline Total & $\mathbf{5 9}$ & $\mathbf{1 0 0}$ \\
\hline
\end{tabular}

Quando comparamos o quadro de áreas temáticas com a fração de enfermeiros assistentes autores destes trabalhos, observamos que há uma produção significativa na área assistencial que não é proporcional à fração desses autores (Tabela 3). Essa categoria foi responsável por $31 \%$ dos trabalhos analisados. Isso indica que outras categorias e não apenas os enfermeiros/ assistentes estão envolvidos com estudos na temática assistência. Os dados mostram, no entanto, que a maioria dos autores é composta por discentes e docentes (69\%). A maior produção na área acadêmica pode ser atribuída a aspectos das funções exercidas pelos docentes, em especial das instituições públicas, onde a ascensão de carreira universitária está relacionada a diversos fatores, tais como, titulação, orientação e publicação. Também a manutenção dos conceitos nos programas de pósgraduação requer publicações de trabalhos de pesquisa ${ }^{(12)}$ o que envolve o corpo docente e discente.

\section{Origem das Publicações}

A origem das publicações em relação à região onde a instituição dos autores está localizada está indicada na Figura 3. Predominam os trabalhos originados na região sudeste enquanto aqueles da região centro-oeste representam são a minoria (63\% versus $4 \%$ ). Esta redominância da região sudeste pode ser justificada pela concentração de escolas de enfermagem, de hospitais e clínicas e de cursos de pós-graduação nesta região.

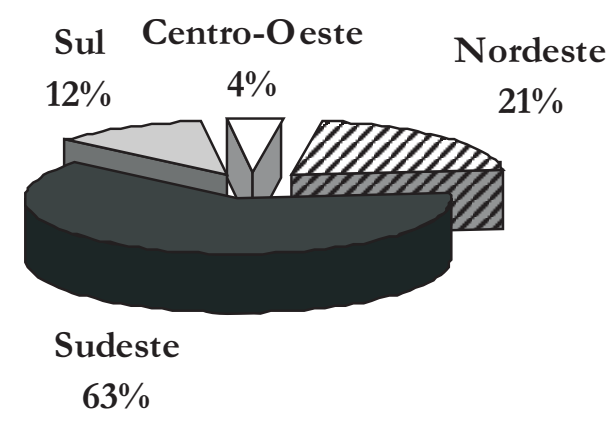

Figura 2- Distribuição da Produção de enfermagem em HHTMO, no Brasil, por região, no período de 20002004.(Percentual calculado sobre o total de identificações $n=77$ )

\section{DISCUSSÃO}

Ainda que os dados apresentados apontem para uma produção reduzida sobre pesquisa de enfermagem em hematologia, hemoterapia e transplante de medula óssea, faz-se necessário um estudo mais detalhado, para melhor entender as causas deste problema. Acreditamos que, dentre alguns outros fatores, a baixa produção possa ser justificada pelo fato de se tratar de uma especialidade da enfermagem ainda recente e não consolidada no Brasil. Já a prevalência de publicações em anais e congressos foi também observada e discutida por Costa 
et al. ${ }^{(13)}$. Sabemos, no entanto, que esta é uma fonte de informação de pouca abrangência e não garante que $\mathrm{o}$ conhecimento gerado alcance todas as comunidades científicas. Para Mancia et al. ${ }^{(14)}$ alguns fatores podem justificar tal prevalência, tais como: (1) produção fora dos padrões estabelecidos, portanto, não considerado "publicável"; (2) pesquisas para responder ao interesse pessoal, circunscrito à própria instituição, sem uma visão de expansão do conhecimento e (3) carência de estratégias de apoio e aperfeiçoamento de pesquisadores iniciantes, que acabam por não divulgar seus trabalhos.

Apesar de algumas limitações, este estudo oferece subsídio para descrever um perfil da produção científica de enfermagem na área de HHTMO. A interpretação dos dados mostra que a produção da área ainda é: (a) incipiente, (b) caracteriza-se, principalmente, por ser uma produção estável ao longo dos últimos anos, escrita em anais de congressos e por discentes e docentes, (c) são originados principalmente na região sudeste e (d) predominam aqueles com enfoque qualitativo e na área assistencial.

No contexto das estratégias facilitadoras da divulgação, é importante destacar o papel dos cursos de pós-graduação em enfermagem. Estes cursos têm como objetivo, especialmente nas modalidades de mestrado e doutorado, formar pesquisadores. Também são avaliados e conceituados pela Coordenação de Aperfeiçoamento de Pessoal de Nível Superior (CAPES), levando em consideração, entre outros fatores, a produção e divulgação científicas de seus discentes e docentes. No entanto, o número de cursos de pósgraduação em enfermagem ainda é muito limitado, dadas as dimensões continentais e as demandas sociais do Brasil. Além disso, é fator agravante que eles se encontram concentrados na região sudeste e muitos estados não apresentam nenhum curso registrado na $\mathrm{CAPES}^{(15)}$. O quadro apresentado neste estudo, de certa forma, corrobora com este cenário nacional e sugere, também, grandes dificuldades do acesso de profissionais à qualificação e habilidade científica em determinadas regiões do país.

\section{CONSIDERAÇÕES FINAIS}

Esperamos que esse trabalho sirva de incentivo à discussão sobre a produção e divulgação científica desta área do conhecimento. Acreditamos que o desenvolvimento científico da área contribuirá para a consolidação da profissão de enfermagem, ajudando a mesma a galgar os degraus necessários para alcançar o status e valorização profissional, frente à sociedade e demais profissões. Contudo, é recomendável que em estudos futuros sejam desenvolvidas análises com um recorte temporal mais amplo, com a inclusão de outros periódicos de enfermagem, em especial dos que disponibilizam os artigos completos por meio de acesso pela Internet, tais como, Acta Paulista de Enfermagem, Online Brazilian
Journal of Nursing, Revista Mineira de Enfermagem e Revista Paulista de Enfermagem. Concordamos com Barros et al. ${ }^{(16)}$ que afirmam que a enfermagem dos países em desenvolvimento deve constituir a sua própria base de dados, face às dificuldades dos indexadores internacionais se adequarem às particularidades destes países.

\section{REFERÊNCIAS}

1. Polit DF, Hungler BP. Introduction to nursing practice. In: PolitDF, Hungler BP. Nursing research: principles and methods. 6th ed. Philadelphia: Lippincott; c1999. Chapter I. pp. 3-17.

2. Carvalho EC. A produção do conhecimento de enfermagem. Rev Latinoam Enfermagem. 1998; 6(1): 119-22.

3. Instituto Nacional de Estudos e Pesquisas Anísio Teixeira. Cursos Presenciais em Enfermagem [texto na Internet]. Brasília (DF): INEP; c2003. [citado 2006 Jul 12] Disponível em: http://www.inep.gov.br .

4. Ministério da Educação. Conselho Nacional de EducaçãoCNE [sítio na Internet].Brasília (DF): Ministério da Educação; 2003. [citado 2006 Jul 12]. Disponível em: http:/ /portal.mec.gov.br/cne/.

5. Ministério da Ciência e Tecnologia. Diretório de Grupos de Pesquisa do Brasil. Senso 2002 [texto na Internet]. Brasília (DF): CNPq; s.d. [citado 2006 Jul 12]. Disponível em: http:/ /dgp.cnpq.br/censo2004/.

6. Instituto Nacional do Cancer (INCA). Atlas da Mortalidade de Câncer no Brasil [texto na Internet]. Brasília (DF): INCA; c1996-2006 . [citado 2006 Jul 12]. Disponível em: http:/ / www.inca.gov.br/atlas/.

7. Narin F, Olivastro D, Stevens KA. Bibliometrics - Theory, practice and problems. Eval Rev. 1994; 18(1): 65-76.

8. Okubo Y. Bibliometric indicators and analysis of research systems: methods and examples. STI Working Papers 1997/ 1. Paris: OCDE/GD; 1997.

9. Leta J,Brito $\mathrm{CH}$. A produção científica brasileira. In: Viotti EB, Macedo MM, organizadores. Indicadores de ciência, tecnologia e inovação no Brasil. Campinas, SP: Editora da UNICAMP; c2003.

10. Assis MMA, Nascimento MAA, Jorge MSB, Matos MS, Braga VAB, Moreira ASP, Garcia TR. Produção científica de enfermagem na Região Nordeste: 1988-1992. Rev Latinoam Enfermagem. 1993; 1 (N Esp): 85-102.

11. Pincus HA, Henderson B, Bladwood D, Dial T. Trends in research in two general psychiatric journals in 1969-1990: research on research. Am J Psychiatry. 1993; 150(1): 135-42.

12. Burlamaque CS. Publicações e difusão do conhecimento em enfermagem no Brasil. Rev Bras Enfermagem. 1987; 40(4): 241-5.

13. Costa RS, Carvalho DV. Análise da produção científica dos enfermeiros de Minas Gerais publicada em periódicos de enfermagem. Rev Latinoam Enfermagem. 2001; 9(5): 19-25.

14. Mancia JR, Ramos FRS. A situação dos periódicos de enfermagem no Brasil. In: Congresso Brasileiro de Enfermagem, 2001, Recife. Congresso Brasileiro de Enfermagem. Recife: Associação Brasileira de Enfermagem (PE); 2000.

15. CAPES. Coordenação de Aperfeiçoamento de Pessoal de Nível Superior [sítio na internet]. Em: http:// www.capes.gov.br/capes/portal/.

16. Barros ALBL, Gutierrez MGR, Michel JLM. Índices cienciométricos e a ciência de enfermagem brasileira [editorial]. Acta Paul Enfermagem. 2005; 18(4): v-vi. 